\title{
Complete genome sequence of Enterococcus durans KLDS6.0933, a potential probiotic strain with high cholesterol removal ability
}

\author{
Bailiang Li ${ }^{1}$, Smith Etareri Evivie ${ }^{1,2}$, Da Jin ${ }^{1}$, Yueyue Meng ${ }^{1}$, Na Li ${ }^{1}$, Fenfen Yan ${ }^{1}$, Guicheng Huo ${ }^{1,3^{*}}$ and Fei Liu ${ }^{1 *}$
}

\begin{abstract}
Background: Enterococci are commensal bacteria in the mammalian gastrointestinal tract which play an important role in the production of various fermented foods. Thus, certain enterococcal strains are commonly used as probiotics to confer health benefits to human and animals. Enterococcus durans KLDS6.0933 is a potential probiotic strain with high cholesterol removal ability, which was isolated from traditional naturally fermented cream in Inner Mongolia of China. To better understand the genetic basis of the probiotic properties of this strain, the whole-genome sequence was performed using the PacBio RSIl platform.

Results: Enterococcus durans KLDS6.0933 contains a circular chromosome of 2,867,028 bp, two plasmids of $163,286 \mathrm{bp}$ and $41,490 \mathrm{bp}$, respectively. Within the 2704 predicted genes, genes involved with acid, bile and oxidative stress resistance were identified. Bile salt hydrolase (BSH, LIANG_RS13510), a cholesterol removal enzyme identified in the E. durans KLDS6.0933 genome is different from that of other Enterococcus strains. Furthermore, unlike other Enterococcus strains, E. durans KLDS 6.0933 can facilitate the complete biosynthesis pathway of L-tryptophan.
\end{abstract}

Conclusions: In silico analysis confirmed the probiotic properties of E. durans KLDS6.0933 and may help us exploit the potential applications of E. durans KLDS6.0933 as an industrially important strain.

Keywords: Enterococcus durans, Genome, Probiotic, Stress, L-Tryptophan, Cholesterol removal

\section{Introduction}

Enterococci are Gram-positive lactic acid bacteria (LAB) and comprise 54 species [1], which are ubiquitously present in the environment, food and the gastrointestinal tracts of diverse hosts. Enterococci may have important roles in various fermented food as they contribute to the sensory properties and ripening of sausages or certain cheeses, presumably through proteolysis, lipolysis, and citrate utilization $[2,3]$. As a prominent member of normal flora, Enterococci play a helpful part in the balance between the gut microbiota and the host.

\footnotetext{
*Correspondence: guichenghuo@126.com; david.as@163.com ${ }^{1}$ Key Laboratory of Dairy Science, Ministry of Education, Northeast Agricultural University, Harbin 150030, People's Republic of China Full list of author information is available at the end of the article
}

Enterococci are commonly used as probiotics to confer health benefits to human and animals. These bacteria can be used in the treatment of irritable bowel syndrome and antibiotic-associated diarrhea in humans as well as in lowering cholesterol levels or regulating immune system to improve health [4-7]. Likewise, the antioxidant potential of Enterococci has been studied [8].

Enterococcus durans KLDS6.0933 was originally isolated from traditional naturally cream samples collected in Inner Mongolia of China. It has been demonstrated that $E$. durans KLDS6.0933 had the potential to resist acid and bile salt, and assimilate cholesterol in a recent in vitro study [6]. In order to analyze these characteristics and mine probiotic properties of this strain from genomic insights, the whole-genome sequence of $E$. durans KLDS6.0933 was carried out and analyzed in 
silico. Comparison of genomic data from $E$. durans KLDS6.0933 with other Enterococcus strains may improve our understanding of the traits of $E$. durans KLDS6.0933.

\section{Methods \\ Strain isolation and DNA extraction \\ Enterococcus durans KLDS6.0933 was isolated from traditional naturally fermented cream in Inner Mon- golia of China and was available at the Key Laboratory of Dairy Science (KLDS), Northeast Agricultural Uni- versity (NEAU), Harbin, China. Before use, E. durans KLDS6.0930 was activated through three propagation steps in M17 broth (Oxoid Ltd, Hampshire, UK) at $37^{\circ} \mathrm{C}$ for $24 \mathrm{~h}$. The genomic DNA of E. durans KLDS6.0933 was extracted using the DNeasy Tissue kit (Qiagen, Ger- many) following the manufacturer's instruction.}

\section{Genome sequencing, assembly, and analysis}

The quantity and purity of total DNA were determined by $2 \%$ agarose gel electrophoresis and a NanoDrop ${ }^{\mathrm{TM}}$ spectrophotometer. The whole-genome sequence of E. durans KLDS6.0933 was carried out on the single molecule real-time by the Pacbio RSII platform (Pacific Biosciences, USA). A $20 \mathrm{~K}$ template library was generated and sequenced by P4-C 2 chemistry on two cells. The raw data was obtained as 59,078 pair-end reads (484 MB) with an average read length of $8198 \mathrm{bp}$. The filtered paired-end reads were de novo assembled by using the hierarchical genome assembly process protocol version 3.0 and polished using Quiver [9]. Gene annotation was determined by Annotation NCBI Prokaryotic Genome Annotation Pipeline [10]. Ribosomal RNA genes were identified using RNAmer 1.2 [11] and tRNA genes were detected using tRNAscan SE v. 2.0 [12]. Functional categories of coding sequences (CDSs) were classified by WebMGA, using RPSBLAST program (applied threshold $1 \mathrm{e}-5)$ for clusters of orthologous groups (COG) annotation [13]. The circular genomic map was constructed using CGView Server [14]. Functional annotation was performed with the Kyoto Encyclopedia of Genes and Genomes (KEGG) database using Bi-directional Best Hit method by KAAS [15] for analyzing L-tryptophan biosynthetic pathway. Using the BSH sequence from the complete genomes of representative strains, a phylogenetic tree was constructed using MEGA 7.0 software with the neighbor-joining method [16]. Comparative genomic analysis was performed between $E$. durans KLDS6.0933 and other representative Enterococcus strains that are relatively close to $E$. durans KLDS6.0933 based on the phylogenetic tree of $16 \mathrm{~S}$ rRNA gene.

\section{Quality assurance}

The genomic DNA used for sequencing was isolated from a single colony of the $E$. durans KLDS6.0933. The $16 \mathrm{~S}$ rRNA gene was sequenced and BLAST was conducted against the NCBI database, then the phylogenetic tree based on the 16S rRNA was constructed by MEGA 7.0 software with the Neighbour-joining method. The result clearly indicated this strain belonged to the species E. durans (Additional file 1: Figure S1). In addition, the average nucleotide identity (ANI) of the genomic sequences between $E$. durans KLDS6.0933 and E. durans ATCC6056 was evaluated by the ANI calculator using the OrthoANIu algorithm at the genomic level. Here, we reported that the value of their ANI was 99.66\% (Additional file 1: Table S1).

\section{Results and discussion General features}

As shown in Fig. 1, the complete genome of $E$. durans KLDS6.0933 is composed of a 2,867,028 bp chromosome with GC content of $38 \%$ and two plasmids-a plasmid of $163,286 \mathrm{bp}$ with a GC content of $35.5 \%$ and another plasmid of $41,490 \mathrm{bp}$ with a GC content of $35.3 \%$. Among the 2704 predicted genes, 2393 CDSs, 86 RNAs and 225 pseudogenes were found in the chromosome of E. durans KLDS6.0933 (Additional file 1: Table S2). Of the identified CDSs, 2024 genes can be classified into COG classes (Additional file 1: Figure S2). The highest number of genes in this strain was found in the functional groups related to carbohydrate metabolism (212).

\section{Identification of genes coding stress resistance and cholesterol removal}

A recent study reported that $E$. durans KLDS6.0933 was highly tolerant to acid [6]. In order to mine the genetic elements contributing to acid tolerance, proton motive force $\mathrm{F}_{1} \mathrm{~F}_{0}$ ATPase subunits, $\mathrm{Na}^{+} / \mathrm{H}^{+}$antiporters, $\mathrm{K}^{+}$ uptake transporter and cation-transporting ATPase were analyzed in the genome of $E$. durans KLDS6.0933, E. durans KLDS6.0933 possesses these genes by facilitating the counteraction acidic stress (Additional file 1: Table S3). Furthermore, probiotics could increase the intracellular $\mathrm{pH}$ via amino acid decarboxylation-antiporter reactions [17], E. durans KLDS6.0933 harbors the tyramine cluster which consists of tyrosine decarboxylase, tyrosine tyramine antiporter and tyrosyltRNA synthetase gene, which decarboxylate tyrosine to improve the acid tolerance (Additional file 1: Table S3). Another mechanism of acid tolerance is the production of ammonia (alkaline compounds) by the arginine deiminase (ADI) pathway, which includes ADI, ornithine 


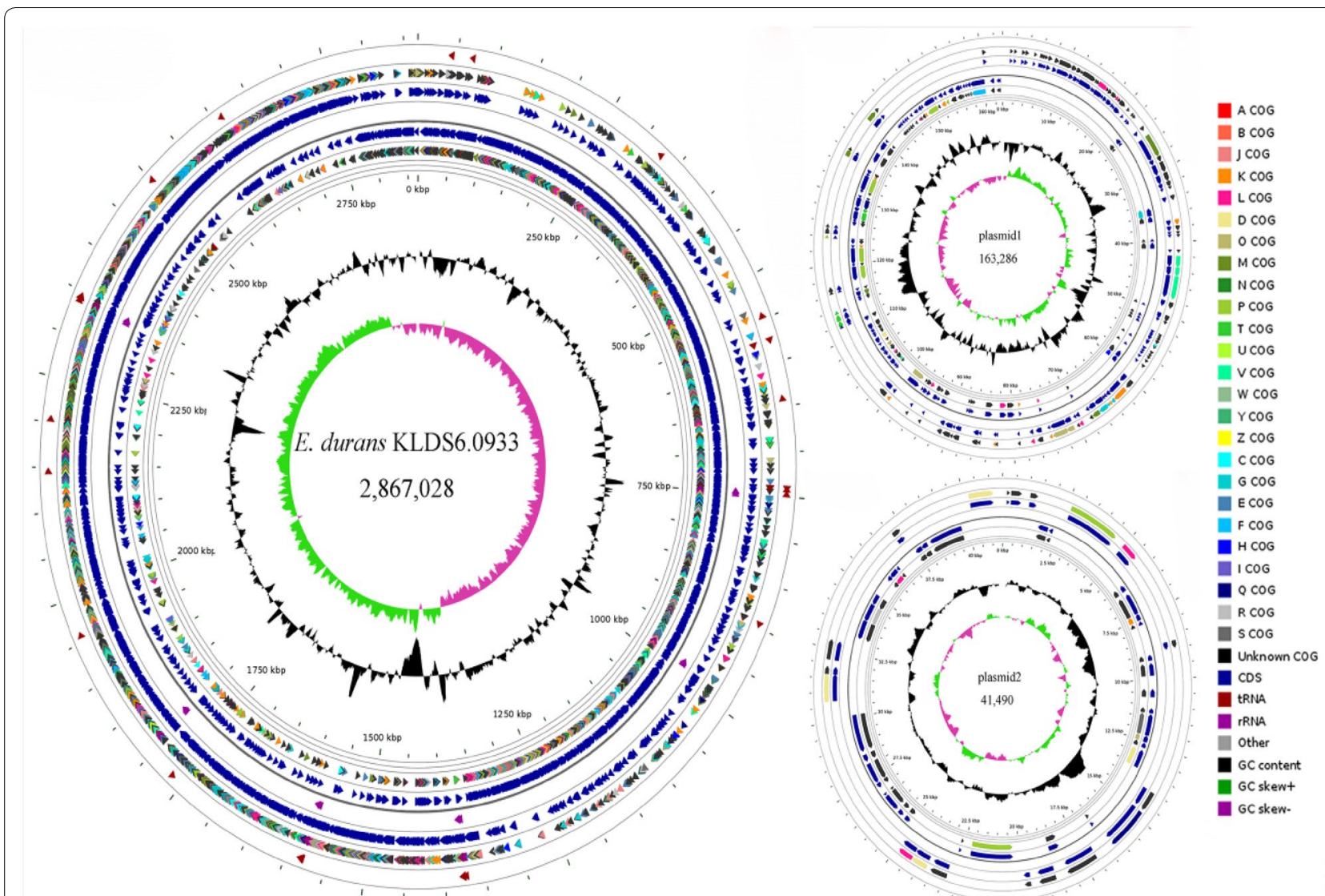

Fig. 1 Circular genome map of Enterococcus durans KLDS6.0933. From periphery to center: tRNA; Protein coding genes (CDSs) on forward strand colored according to the assigned COG classes; Genes on forward strand; rRNA; Genes on reverse strand; CDSs on reverse strand colored according to the assigned COG classes; Genome position in kbp; GC content; $G C$ skew $(G-C) /(G+C)$

transcarbamylase, carbamate kinase and arginine-ornithine transporter [18]. These genes are presented in $E$. durans KLDS6.0933 (Additional file 1: Table S3).

The effect of bile on the growth of $E$. durans KLDS6.0933 showed that this strain had bile tolerance property [6], a gene encoding BSH (LIANG_RS13510), a member of cholylglycine hydrolase family, was identified in the genome of $E$. durans KLDS6.0933, which catalyzes the hydrolysis of glycine- and taurine-conjugated bile salts into amino acid residues and free bile acids [19]. Bile salt deconjugation by this enzyme can lower serum cholesterol level [20]. Supporting this, E. durans KLDS6.0933 showed high cholesterol removal ability in our previous study [6]. Phylogenetic relationship among the selected BSH sequences of $E$. durans KLDS6.0933, E. canis DSM17029, E. faecium 6E6, E. hirae R17, E. mundtii EMB156, E. ratti DSM15687, E. thailandicus a523 and $E$. villorum ATCC700913 that had more homology in $16 \mathrm{~S}$ RNA gene with each other were represented on a neighbor-joining tree, which was constructed using amino acid sequences of BSH with bootstrap replication of 1000 in
MEGA 7.0 software. Phylogenetic tree analysis (Fig. 2a) showed that the BSH (LIANG_RS13510) of E. durans KLDS 6.0933 is more closely related to that of $E$. mundtii EMB156 than other Enterococcus strains. However, they are still evolutionarily distant. In addition, alignment and comparison of BSH sequences and their contexts were studied using BLASTp, as shown in Fig. 2b. We found that BSH sequences showed low sequence identities with that of E. durans KLDS6.0933 and the contexts of BSH sequences were different, these imply that $\mathrm{BSH}$ is specific to genus and it is significant to further study the relationship between gene structure and enzymatic activity of $\mathrm{BSH}$.

\section{Identification of genes coding antioxidant system}

Oxidative stress occurs when abnormally high levels of reactive oxygen species (ROS) are generated, resulting in nucleic acid, protein and lipid damage [21]. The antioxidant mechanisms of probiotics were associated with enzymatic and non-enzymatic antioxidative system. Among enzymatic antioxidative system, the most 


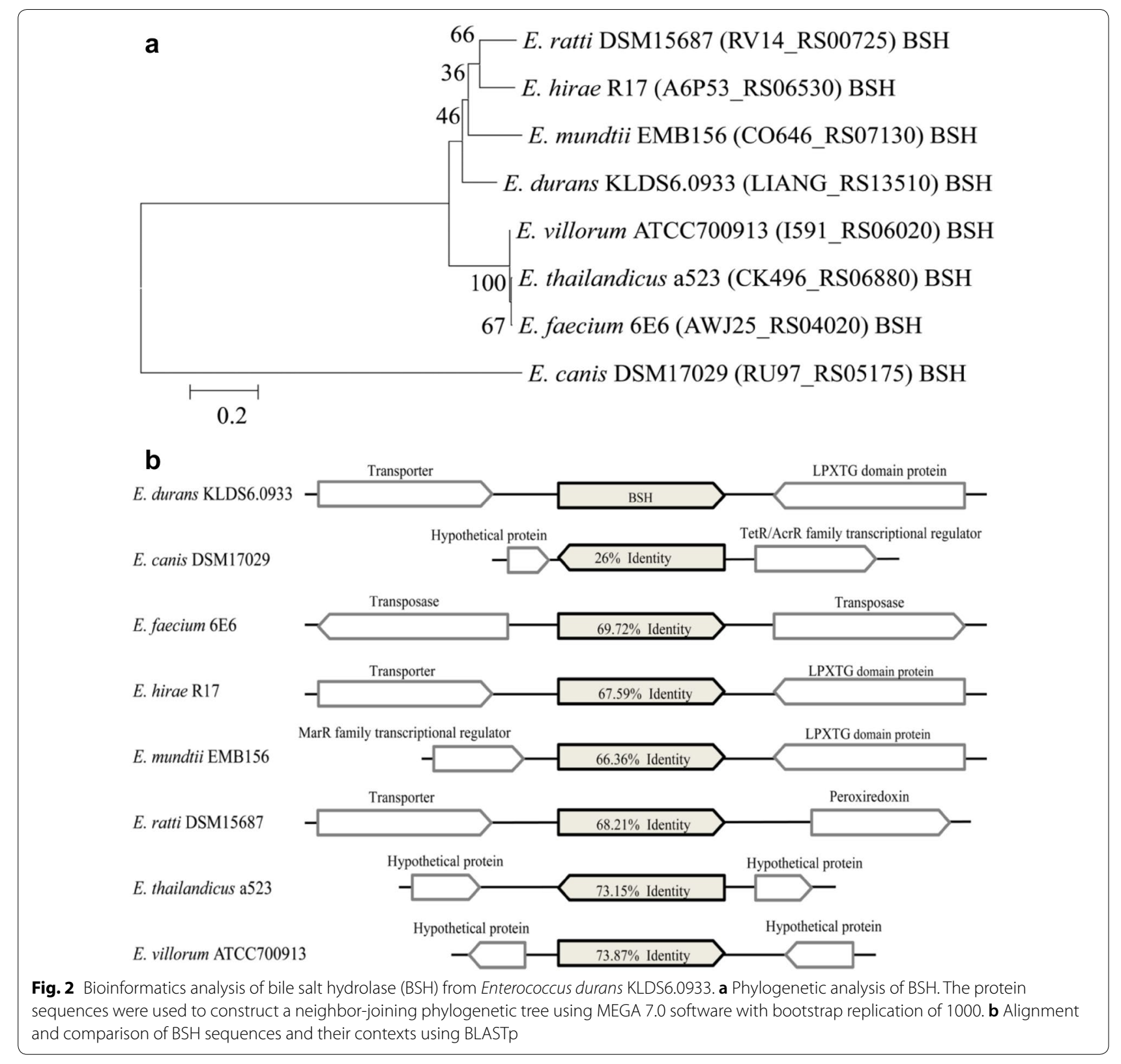

conserved oxidative resistance mechanism in the $\mathrm{LAB}$ is that oxygen is reduced indirectly to water by coupling of NADH oxidase and NADH peroxidase oxidative [22]. Another important player in resistance to oxidative stress is superoxide dismutase (SOD), which scavenges superoxide anion radicals [21]. Bacteria can also counteract the negative effects of oxidation through catalase, an enzyme catalyzing the detoxification of $\mathrm{H}_{2} \mathrm{O}_{2}$ [23]. Genomic insights into antioxidant activity revealed that the genes encoding NADH oxidase, NADH peroxidase, SOD, catalase and glutathione peroxidase were found in the genome of $E$. durans KLDS6.0933 (Table 1), which are the main components of ROS resistome in the LAB. To cope with oxidative stress, $E$. durans KLDS6.0933 carries diverse genes as shown in Table 1 , namely methionine sulfoxide reductase, $S$-methyltransferase, $S$-ribosyl homocysteinase and $S$-adenosylmethionine synthetase, alkyl hydroperoxide reductase and organic hydroperoxide reductase [24].

The non-enzymatic antioxidative system has been suggested to be mainly composed of mercapto peptides and regulators. E. durans KLDS6.0933 harbors complete glutathione and thioredoxin systems. The presence of glutathione reductase, glutathione-disulfide reductase, 
Table 1 Putative genes for antioxidative response in Enterococcus durans KLDS6.0933

\begin{tabular}{|c|c|}
\hline Encoded protein & Locus tag(s) \\
\hline NADH oxidase & LIANG_RS12680 \\
\hline NADH peroxidase & LIANG_RS04555 \\
\hline Manganese superoxide dismutase & LIANG_RS10670 \\
\hline Manganese catalase & LIANG_RS08960 \\
\hline \multirow[t]{2}{*}{ Methionine sulfoxide reductase } & $\begin{array}{l}\text { LIANG_RS06665, LIANG_RS07790, } \\
\text { LIANG_RS11590 }\end{array}$ \\
\hline & LIANG_RS05685 \\
\hline S-Adenosylmethionine synthetase & LIANG_RS07580 \\
\hline $\begin{array}{l}\text { S-Adenosylmethionine ribosyl- } \\
\text { transferase }\end{array}$ & LIANG_RS05360 \\
\hline S-Ribosyl homocysteinase & LIANG_RS04630 \\
\hline Alkyl hydroperoxide reductase & LIANG_RS00005 \\
\hline Organic hydroperoxide reductase & LIANG_RS13495 \\
\hline Glutathione peroxidase & LIANG_RS04635 \\
\hline Glutathione reductase & LIANG_RS11780 \\
\hline Glutathione-disulfide reductase & LIANG_RS06555 \\
\hline Thioredoxin & LIANG_RS01070 \\
\hline Thioredoxin-disulfide reductase & LIANG_RS02305 \\
\hline Thiol reductase thioredoxin & $\begin{array}{l}\text { LIANG_RS02900, LIANG_RS03115, } \\
\text { LIANG_RS08005 }\end{array}$ \\
\hline $\begin{array}{l}\text { Redox-sensing transcriptional } \\
\text { repressor Rex }\end{array}$ & LIANG_RS03455, LIANG_RS10045 \\
\hline DNA-binding ferritin-like protein & LIANG_RS06680, LIANG_RS08210 \\
\hline \multirow[t]{4}{*}{ Stress protein } & $\begin{array}{l}\text { LIANG_RS08010, LIANG_RS08315, } \\
\text { LIANG_RS08700 }\end{array}$ \\
\hline & $\begin{array}{l}\text { LIANG_RS00090, LIANG_RS00170, } \\
\text { LIANG_RS00205 }\end{array}$ \\
\hline & $\begin{array}{l}\text { LIANG_RS02415, LIANG_RS03580, } \\
\text { LIANG_RS05495 }\end{array}$ \\
\hline & $\begin{array}{l}\text { LIANG_RS11215, LIANG_RS02410, } \\
\text { LIANG_RS12370 }\end{array}$ \\
\hline
\end{tabular}

thioredoxin, thiol reductase thioredoxin and thioredoxin-disulfide reductase in the genome confers it with antioxidant capacity (Table 1). Regulators and stress proteins contribute to triggering different stress responses to protect against oxidative damage when bacterial cells encounter a specific stress condition [22]. These genes were found in the genome of E. durans KLDS6.0933 (Table 1).

\section{L-Tryptophan biosynthesis pathway}

Comparison of the genomes of E. durans KLDS6.0933, E. canis DSM17029, E. faecium 6E6, E. hirae R17, E. mundtii EMB156, E. ratti DSM15687, E. thailandicus a523 and E. villorum ATCC700913 revealed that only $E$. durans KLDS6.0933 can synthesize L-tryptophan, which

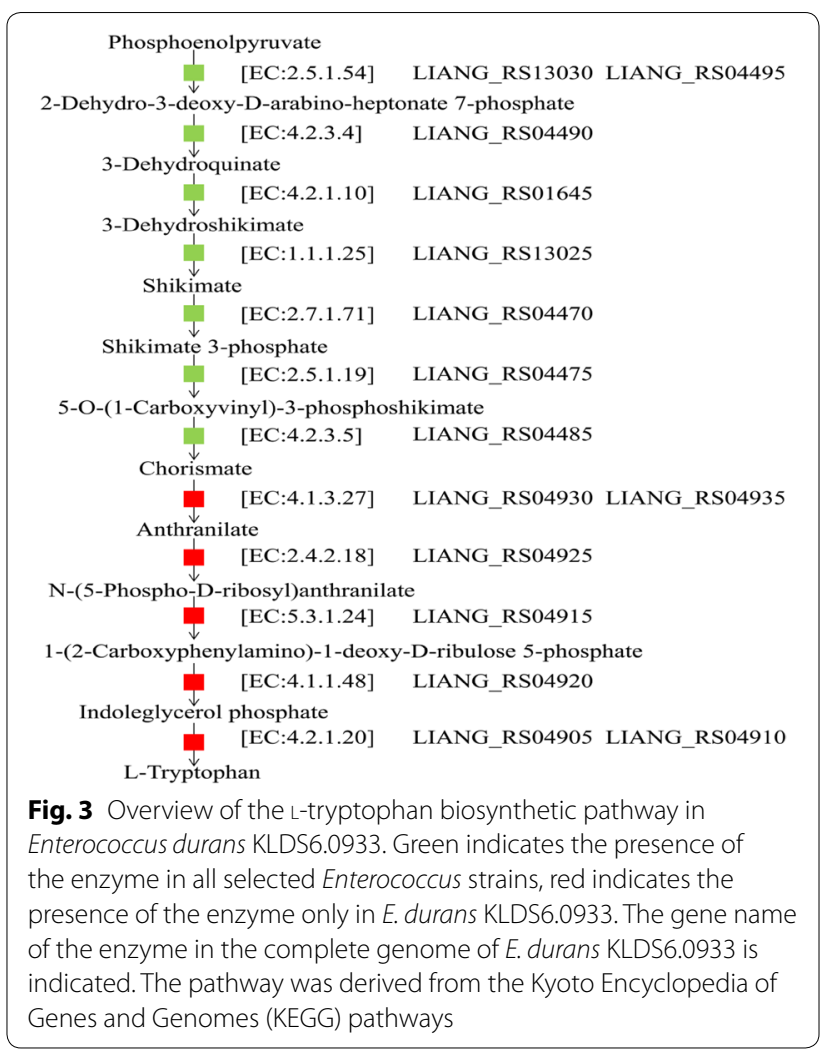

is an essential amino acid for humans and other animals and widely used in food, animal feed, and pharmaceutical industries $[25,26]$. E. durans KLDS6.0933 presents a complete L-tryptophan biosynthetic pathway (Fig. 3) and uses phosphoenolpyruvate as an intermediate, which can be formatted from the pathway of glycolysis. The part of this gene set was also found in other selected Enterococcus strains, but these strains lack genes encoding anthranilate synthase, anthranilate phosphoribosyltransferase, phosphoribosylanthranilate isomerase, indole-3-glycerol phosphate synthase and tryptophan synthase, which are essential for producing L-tryptophan from chorismate. Furthermore, the genome of E. durans KLDS6.0933 does not carry any genes related to the L-tryptophan degradation pathway, thus indicating that E. durans KLDS6.0933 can biosynthesize L-tryptophan de novo.

In conclusion, the complete genome sequence of $E$. durans KLDS6.0933 allows us to better understand the genetic basis of its probiotic potentials. These data will help us explore its potential applications as an important strain in the food industry. However, more in vivo and in vitro researches need to be done to verify the probiotic properties and evaluate its safety status. 


\section{Additional file}

Additional file 1: Figure S1. Neighbour-joining tree based on the 165 rRNA gene sequences of strain KLDS6.0933 and phylogenetically related Enterococcus strains. Bootstrap values based on 1000 resampled datasets are shown at branch nodes. Figure S2. Clusters of orthologous groups (COG) functional categories in the complete genome of Enterococcus durans KLDS6.0933. Table S1. Average nucleotide identity (ANI) of the genomic sequences between Enterococcus durans KLDS6.0933 and Enterococcus durans ATCC6056. Table S2. General genome features of Enterococcus durans KLDS6.0933. Table S3. Putative genes for acid stress response in Enterococcus durans KLDS6.0933.

\section{Abbreviations}

BSH: bile salt hydrolase; LAB: lactic acid bacteria; COG: clusters of orthologous groups; KEGG: Kyoto Encyclopedia of Genes and Genomes; CDSs: coding sequences; ANI: average nucleotide identity; ADl: arginine deiminase; ROS: reactive oxygen species.

\section{Authors' contributions}

GH and BL designed the study; BL, DJ, FL, FY, YM and NL analyzed data; SM and $B L$ wrote the manuscript. All authors read and approved the final manuscript.

\section{Author details}

${ }^{1}$ Key Laboratory of Dairy Science, Ministry of Education, Northeast Agricultural University, Harbin 150030, People's Republic of China. ${ }^{2}$ Food Science and Nutrition Unit, Department of Animal Science, Faculty of Agriculture, University of Benin, PMB 1154, Benin City, Nigeria. ${ }^{3}$ Food College, Northeast Agricultural University, Harbin 150030, People's Republic of China.

\section{Acknowledgements}

Not applicable.

\section{Competing of interests}

The authors declare that they have no competing interests.

\section{Availability of data and materials}

The completed genome sequence has been deposited in GenBank database with accession number CP012366.1 (chromosome), CP012367.1 (plasmid 1) and CP012368.1 (plasmid 2), respectively.

\section{Consent for publication}

Not applicable.

\section{Ethics approval and consent to participate}

Not applicable.

\section{Funding}

This research was supported by the National Key Research and Development Program of China (Grant No. 2017YFD0400303).

\section{Publisher's Note}

Springer Nature remains neutral with regard to jurisdictional claims in published maps and institutional affiliations.

Received: 10 May 2018 Accepted: 16 July 2018

Published online: 19 July 2018

\section{References}

1. Parte AC. LPSN - list of prokaryotic names with standing in nomenclature. Nucleic Acids Res. 2014;42:613-6.

2. Moreno MFR, Sarantinopoulos P, Tsakalidou E, Vuyst L. The role and application of enterococci in food and health. Int J of Food Microbiol. 2006;106:1-24.
3. Bhardwaj A, Malik RK, Chauhan P. Functional and safety aspects of enterococci in dairy foods. Indian J of Microbiol. 2008;48:317-25.

4. Bybee SN, Scorza AV, Lappin MR. Effect of the probiotic Enterococcus faecium SF68 on presence of diarrhea in cats and dogs housed in an animal shelter. J Vet Int Med. 2011;25:856.

5. Cao GT, Zeng XF, Chen AG, Zhou L, Zhang L, Xiao YP, Yang CM. Effects of a probiotic, Enterococcus faecium, on growth performance, intestinal morphology, immune response, and cecal microflora in broiler chickens challenged with Escherichia coli K88. Poultry Sci. 2013;92:2949.

6. Guo L, Li T, Tang Y, Yang L, Huo G. Probiotic properties of Enterococcus strains isolated from traditional naturally fermented cream in China. Microb Biotechnol. 2016;9:737-45.

7. Franz CM, Huch M, Abriouel H, Holzapfel W, Gálvez A. Enterococci as probiotics and their implications in food safety. Int J Food Microbiol. 2011;151:125-40.

8. Pieniz S, Andreazza R, Anghinoni T, Camargo F, Brandelli A. Probiotic potential, antimicrobial and antioxidant activities of Enterococcus durans strain LAB18s. Food Control. 2014;37:251-6.

9. Chin CS, Alexander DH, Marks P, Klammer AA, Drake J, Heiner C, Clum A, Copeland A, Huddleston J, Eichler EE. Nonhybrid, finished microbial genome assemblies from long-read SMRT sequencing data. Nat Methods. 2013;10:563.

10. Pruitt KD, Tatusova T, Brown GR, Maglott DR. NCBI reference sequences (RefSeq): current status, new features and genome annotation policy. Nucleic Acids Res. 2012;40:130-5.

11. Lagesen $K$, Hallin P, Rødland EA, Staerfeldt HH, Rognes T, Ussery DW. RNAmmer: consistent and rapid annotation of ribosomal RNA genes. Nucleic Acids Res. 2007;35:3100.

12. Lowe TM, Chan PP. tRNAscan-SE On-line: integrating search and context for analysis of transfer RNA genes. Nucleic Acids Res. 2016;44:W54-7.

13. Wu S, Zhu Z, Fu L, Niu B, Li W. WebMGA: a customizable web server for fast metagenomic sequence analysis. BMC Genomics. 2011;12:1-9.

14. Grant JR, Stothard P. The CGView Server: a comparative genomics tool for circular genomes. Nucleic Acids Res. 2008;36:181-4.

15. Moriya Y, Itoh M, Okuda S, Yoshizawa AC, Kanehisa M. KAAS: an automatic genome annotation and pathway reconstruction server. Nucleic Acids Res. 2007;35:182-5.

16. Kumar S, Stecher G, Tamura K. MEGA7: molecular evolutionary genetics analysis version 7.0 for bigger datasets. Mol Biol Evol. 2016;33:1870.

17. Perez M, Calles-Enríquez M, Nes I, Martin MC, Fernandez M, Ladero V, Alvarez MA. Tyramine biosynthesis is transcriptionally induced at low pH and improves the fitness of Enterococcus faecalis in acidic environments. Appl Microbiol Biotechnol. 2015;99:3547-58.

18. Chou L, Weimer BC, Cutler R. Relationship of arginine and lactose utilization by Lactococcus lactis ssp. lactis ML3. Int Dairy J. 2001;11:253-8.

19. Kim GB, Yi SH, Lee BH. Purification and characterization of three different types of bile salt hydrolases from Bifidobacterium strains. J Dairy Sci. 2004;87:258-66.

20. Begley M, Hill C, Gahan CGM. Bile salt hydrolase activity in probiotics. Appl Environ Microbiol. 2006;72:1729.

21. Papadimitriou K, Alegría Á, Bron PA, De AM, Gobbetti M, Kleerebezem M, Lemos JA, Linares DM, Ross P, Stanton C. Stress physiology of lactic acid bacteria. Microbiol Mol Biology Rev (MMBR). 2016;80:837.

22. Miyoshi A, Rochat T, Gratadoux JJ, Le LY, Oliveira SC, Langella P, Azevedo V. Oxidative stress in Lactococcus lactis. Genet Mol Res. 2003;2:348-59.

23. Frankenberg L, Brugna M, Hederstedt L. Enterococcus faecalis hemedependent catalase. J Bacteriol. 2002;184:6351-6.

24. Senan S, Prajapati JB, Joshi CG. Whole-genome based validation of the adaptive properties of Indian origin probiotic Lactobacillus helveticus MTCC 5463. J Sci of Food Agric. 2015;95:321-8.

25. Ikeda M. Towards bacterial strains overproducing L-tryptophan and other aromatics by metabolic engineering. Appl Microbiol Biotechnol. 2006;69:615.

26. Leuchtenberger W, Huthmacher K, Drauz K. Biotechnological production of amino acids and derivatives: current status and prospects. Appl Microbiol Biotechnol. 2005;69:1-8. 